Pacific Journal of Mathematics

EIGENVALUES OF THE ADJACENCY MATRIX OF CUBIC 


\title{
EIGENVALUES OF THE ADJACENCY MATRIX OF CUBIC LATTICE GRAPHS
}

\section{RENU LASKAR}

\begin{abstract}
A cubic lattice graph is defined to be a graph $G$, whose vertices are the ordered triplets on $n$ symbols, such that two vertices are adjacent if and only if they have two coordinates in common. If $n_{2}(x)$ denotes the number of vertices $y$, which are at distance 2 from $x$ and $A(G)$ denotes the adjacency matrix of $G$, then $G$ has the following properties: $\left(\mathbf{P}_{1}\right)$ the number of vertices is $n^{3} .\left(\mathbf{P}_{2}\right) G$ is connected and regular. $\left(\mathbf{P}_{3}\right) n_{2}(x)=3(n-1)^{2} .\left(\mathbf{P}_{4}\right)$ the distinct eigenvalues of $A(G)$ are $-3, n-3,2 n-3,3(n-1)$. It is shown here that if $n>7$, any graph $G$ (with no loops and multiple edges) having the properties $\left(P_{1}\right)-\left(P_{4}\right)$ must be a cubic lattice graph. An alternative characterization of cubic lattice graphs has been given by the author (J. Comb. Theory, Vol. 3, No. 4, December 1967, 386-401).
\end{abstract}

We shall consider only finite undirected graphs without loops or multiple edges. A cubic lattice graph with characteristic $n$ is defined to be a graph whose vertices are identified with the $n^{3}$ ordered triplets on $n$ symbols, with two vertices adjacent if and only if their corresponding triplets have two coordinates in common. If $d(x, y)$ denotes the distance between two vertices $x$ and $y$ and $\Delta(x, y)$ the number of vertices adjacent to both $x$ and $y$, then it has been shown by the author [6] that for $n>7$, the following properties characterize the cubic lattice graph with characteristic $n$ :

$\left(b_{1}\right)$ The number of vertices is $n^{3}$.

$\left(b_{2}\right)$ The graph is connected and regular of degree $3(n-1)$.

$\left(b_{3}\right)$ If $d(x, y)=1$, then $\Delta(x, y)=n-2$.

$\left(b_{4}\right)$ If $d(x, y)=2$, then $\Delta(x, y)=2$.

$\left(b_{5}\right)$ If $d(x, y)=2$, there exist exactly $n-1$ vertices $z$, adjacent to $x$ such that $d(y, z)=3$.

Dowling [4] in a note has shown that the property $\left(b_{5}\right)$ is implied by properties $\left(b_{1}\right)-\left(b_{4}\right)$ for $n>7$. Hence for $n>7,\left(b_{1}\right)-\left(b_{4}\right)$ characterize a cubic lattice graph with characteristic $n$.

The adjacency matrix $A(G)$ of a graph $G$ is a square $(0,1)$ matrix whose rows and columns correspond to the vertices of $G$, and $a_{i j}=1$ if and only if $i$ and $j$ are adjacent. Let $n_{2}(x)$ denote the number of vertices $y$ at distance 2 from $x$.

A cubic lattice graph $G$ with characteristic $n$ has the following properties:

$\left(P_{1}\right)$ The number of vertices is $n^{3}$. 
$\left(P_{2}\right) \quad G$ is connected and regular.

$\left(P_{3}\right) \quad n_{2}(x)=3(n-1)^{2}$ for all $x$ in $G$.

$\left(P_{4}\right)$ The distinct eigenvalues of $A(G)$ are $-3, n-3,2 n-3,3(n-1)$. $\left(P_{1}\right),\left(P_{2}\right),\left(P_{3}\right)$ are obvious. $\left(P_{4}\right)$ is proved in paragraph 2 . We go on to show that $\left(P_{1}\right),\left(P_{2}\right),\left(P_{3}\right),\left(P_{4}\right)$ characterize a cubic lattice graph with characteristic $n$. Similar characterization for tethrahedral graphs has been given by Bose and Laskar [1].

2. Determination of the eigenvalues of $A(G)$. Given $v$ objects, a relation satisfying the following conditions is said to be an association scheme with $m$ classes:

(a) Any two objects are either 1 st, 2 nd, $\cdots$, or $m$ th associates, the relation of association being symmetrical.

(b) Each object $\alpha$ has $n_{i} i$ th associates, the number $n_{i}$ being independent of $\alpha$.

(c) If any two objects $\alpha$ and $\beta$ are $i$ th associates, then the number of objects which are $j$ th associates of $\alpha$, and $k$ th associates of $\beta$, is $p_{\jmath k}^{i}$ and is independent of the pair of $i$ th associates $\alpha$ and $\beta$.

The numbers $v, n_{i}$ and $p_{j k}^{i}, i, j, k=1,2, \cdots, m$ are the parameters of the association scheme.

The concept of an association scheme was first introduced by Bose and Shimamoto [3].

If we define

$$
B_{i}=\left(b_{\alpha i}^{\beta}\right)=\left(\begin{array}{cccc}
b_{. i}^{1} & b_{1 i}^{2} & \cdots & b_{1 i}^{v} \\
b_{2 i}^{1} & b_{2 i}^{2} & \cdots & b_{2 i}^{v} \\
\cdots & \cdots & \cdots & \cdots \\
b_{v i}^{1} & b_{v i}^{2} & \cdots & b_{v i}^{v}
\end{array}\right)
$$

$i=0,1,2, \cdots, m$,

where

$b_{\alpha i}^{\beta}=1$, if the objects $\alpha$ and $\beta$ are $i$ th associates $=0$, otherwise,

and

$$
\mathscr{P}_{k}=\left(p_{i k}^{j}\right)=\left(\begin{array}{cccc}
p_{0 k}^{0} & p_{0 k}^{1} & \cdots & p_{0 k}^{m} \\
p^{0}{ }_{k} & p_{1 k}^{1} & \cdots & p_{1 k}^{m} \\
\cdots & \cdots & \cdots & \cdots \\
p_{m k}^{0} & p_{m k}^{1} & \cdots & p_{m k}^{m}
\end{array}\right), \quad k=0,1, \cdots, m,
$$

then it has been shown by Bose and Mesner [2], that the matrices $\mathscr{P}_{i}, i=0,1, \cdots, m$ are linearly independent and combine in the same way as the $B^{\prime}$ s under addition as well as multiplication. It was further shown that if 


$$
\begin{aligned}
B & =\sum_{i=0}^{m} c_{i} B_{i} \\
\mathscr{P} & =\sum_{i=0}^{m} c_{i} \mathscr{P}_{i},
\end{aligned}
$$

then $B$ and $\mathscr{P}$ have the same distinct eigenvalues. If in particular we take $c_{0}=0, c_{1}=1, c_{2}=c_{3}=\cdots=c_{m}=0$, it follows that the distinct eigenvalues of $B_{1}$ are the same as those of $\mathscr{P}_{1}$.

Consider a cubic lattice graph $G$ with characteristic $n$. If a relation of association on the vertices of $G$ is defined, such that two vertices are 1st, 2 nd, or 3 rd associates if they are at distances 1,2 or 3 respectively, then it can be easily checked that $G$ yields a three-class association scheme. It may be pointed out that the matrix $A(G)$ is the matrix $B_{1}$ and thus the distinct eigenvalues of $A(G)$ are given by those of the matrix

$$
\mathscr{P}_{1}=\left(\begin{array}{cccc}
0 & 1 & 0 & 0 \\
n_{1} & p_{11}^{2} & p_{11}^{2} & p_{11}^{3} \\
0 & p_{12}^{1} & p_{12}^{2} & p_{12}^{3} \\
0 & p_{13}^{1} & p_{13}^{2} & p_{13}^{3}
\end{array}\right) .
$$

The parameters $p_{j k}^{i}$ of the association scheme corresponding to $G$ are easily calculated. They are given by

$$
\begin{array}{llll}
n_{1}=3(n-1), & p_{11}^{1}=n-2, & p_{11}^{2}=2, & p_{11}^{3}=0, \\
& p_{12}^{1}=2(n-1), & p_{12}^{2}=2(n-2), & p_{12}^{3}=3, \\
& p_{13}^{1}=0, & p_{13}^{2}=n-1, & p_{13}^{3}=3(n-2) .
\end{array}
$$

Substituting these values in the matrix $\mathscr{P}_{1}$, the eigenvalues are easily calculated. They are found to be

$$
-3, n-3,2 n-3,3(n-1) \text {. }
$$

Thus, we have the following lemma:

LEMMA 2.1. If $G$ is a cubic lattice graph with characteristic $n$ and if $A(G)$ is the adjacency matrix of $G$, then the distinct eigenvalues of $(A) G$ are

$$
-3, n-3,2 n-3,3(n-1) \text {. }
$$

3. Some preliminaries on matrices. Before stating the next lemma, we need the concept of the polynomial of a graph introduced by Hoffman [5]. Let $J$ be the matrix all of whose entries are unity. Then for any graph $G$ with adjacency matrix $A=A(G)$, there exists a polynomial $P(x)$ such that $P(A)=J$ if and only if $G$ is regular and 
connected. The unique polynomial of least degree satisfying this equation is called the polynomial of $G$, and is calculated as follows: if $G$ has $v$ vertices, it is regular of degree $d$, and the other distinct eigenvalues of $A(G)$ are $\alpha_{1}, \alpha_{2}, \cdots, \alpha_{t}$, then

$$
P(x)=\frac{v \prod_{i=1}^{t}\left(x-\alpha_{i}\right)}{\prod_{i=1}^{t}\left(d-\alpha_{i}\right)} .
$$

Consider a regular connected graph $H$ (with no loops and multiple edges) on $v=n^{3}$ vertices such that the adjacency matrix $A=A(H)$ has the distinct eigenvalues $-3, n-3,2 n-3,3(n-1)$.

Lemma 3.1. The matrix $A$ satisfies the equation

$$
\begin{aligned}
& A^{3}-A^{2}(3 n-9)+A\left(2 n^{2}-18 n+27\right) \\
& \quad+\left(6 n^{2}-27 n+27\right) I=6 J,
\end{aligned}
$$

where $J$ is a $v \times v$ matrix all of whose entries are 1 , and $I$ is the $v \times v$ identity matrix.

Proof. It follows immediately by calculating the polynomial of the graph as given in (3.1).

Lemma 3.2. For any two vertices $x, y$ in $H, d(x, y) \leq 3$.

Proof. If in (3.2) we set $A_{i j}=0, A_{i j}^{2}=0$, then $A_{i j}^{3}=6$, but this implies that $d(i, j) \leq 3$ for all vertices $i, j$ in $H$.

Lemma 3.3. Consider the matrix

$$
B=\frac{1}{2}\left\{A^{2}-(n-2) A-3(n-1) I\right\} .
$$

Let $n_{2}(i)$ denote the number of vertices $j$, such that $d(i, j)=2$, and $n_{3}(i)$ denote the number of vertices $k$, such that $d(i, k)=3$. If $n_{2}(i)=$ $3(n-1)^{2}$ for all vertices $i$ in $H$, then

(i) $B$ is a $(0,1)$ matrix,

(ii) $\Delta(x, y)=n-2$, for all vertices $x, y$ in $H$, such that $d(x, y)=1$,

(iii) $\Delta(x, y)=2$, for all vertices $x, y$ in $H$, such that $d(x, y)=2$.

Proof. Since $H$ is regular and $3(n-1)$ is the dominant eigenvalue, it follows $H$ is regular of degree $n_{1}=3(n-1)$.

Divide the set of vertices of $H$, with respect to a particular vertex $i$ into four subsets $S_{0}, S_{1}, S_{2}, S_{3}$ as follows: 
$S_{0}: i$

$S_{1}: j_{1}, j_{2}, \cdots, j_{t} \cdots, j_{n_{1}}$, such that $d\left(i, j_{t}\right)=1, t=1,2, \cdots, n_{1}$

$S_{2}: k_{1}, k_{2}, \cdots, k_{s}, \cdots, k_{n_{2}(i)}$, such that $d\left(i, k_{s}\right)=2, s=1,2, \cdots, n_{2}(i)$

$S_{3}: l_{1}, l_{2}, \cdots, l_{r}, \cdots, l_{n_{3}(i)}$, such that $d\left(i, l_{r}\right)=3, r=1,2, \cdots, n_{3}(i)$. Thus the vertices in $S_{t}$ are $t$ th associates of the vertex $i$. The following relations can be deduced easily from (3.2) by noting that $A J=J A$.

$$
\begin{aligned}
A_{i i}^{3} & =\sum_{t=1}^{n_{1}} A_{i j_{t}}^{2} \\
& =3(n-1)(n-2) . \\
A_{i i}^{4} & =\sum_{t=1}^{n_{1}} A_{i j_{t}}^{3} \\
& =3(n-1)\left(n^{2}+3 n-3\right) .
\end{aligned}
$$

Also, since $A^{t} J=\{3(n-1)\}^{t} J$, we get

$$
\begin{aligned}
\sum_{j=1}^{v} A_{i j}^{2} & =\left(A^{2} J\right)_{i i} \\
& =9(n-1)^{2}, \\
A_{i i}^{2} & =\sum_{t=1}^{n_{1}} A_{i j_{t}} \\
& =3(n-1) .
\end{aligned}
$$

Also

$$
\sum_{r=1}^{n_{3(i)}} A_{i l_{r}}^{2}=0
$$

Hence it follows from (3.3), (3.5), (3.6), (3.7) that

$$
\begin{aligned}
\sum_{s=1}^{n_{2(i)}} A_{i k_{s}}^{2} & =\sum_{j=1}^{v} A_{i j}^{2}-\sum_{t=1}^{n_{1}} A_{i j_{t}}^{2}-\sum_{r=1}^{n_{3(i)}} A_{i l_{r}}^{2}-A_{i \imath}^{2} \\
& =6(n-1)^{2} .
\end{aligned}
$$

Consider

$$
\begin{aligned}
X_{i} & =b_{i i}^{2}+\sum_{t=1}^{n_{1(i)}} b_{i j_{t}}^{2}+\sum_{s=1}^{n_{2(i)}}\left(b_{i k_{s}}-1\right)^{2}+\sum_{r=1}^{n_{3(i)}} b_{i l_{r}}^{2} \\
& =\sum_{j=1}^{v} b_{i j}^{2}-2 \sum_{s=1}^{n_{2(i)}} b_{i k_{s}}+n_{2}(i) .
\end{aligned}
$$

We first show that

$$
X_{i}=n_{2}(i)-3(n-1)^{2}
$$

Since

$$
B=\frac{1}{2}\left[A^{2}-(n-2) A-3(n-1) I\right] \text {, we get }
$$




$$
\begin{gathered}
B_{i i}^{2}=\frac{1}{4}\left[A_{i i}^{4}-2(n-2) A_{i i}^{3}+\left(n^{2}-10 n+10\right) A_{i i}^{2}\right. \\
\left.+6\left(n^{2}-3 n+2\right) A_{i i}+9(n-1)^{2} I_{i i}\right] .
\end{gathered}
$$

Substituting values from (3.3), (3.4), (3.6) in (3.11) we get

$$
B_{i i}^{2}=3(n-1)^{2} \text {. }
$$

But

$$
\sum_{j=1}^{v} b_{i j}^{2}=B_{i i}^{2}
$$

Hence

$$
\sum_{\jmath=1}^{v} b_{i j}^{2}=3(n-1)^{2}
$$

Also from (3.10)

$$
\sum_{s=1}^{n_{2(i)}} b_{i k_{s}}=\frac{1}{2} \sum_{s=1}^{n_{2(i)}} A_{i k_{s}}^{2} .
$$

It follows from (3.8) that

$$
\sum_{s=1}^{n_{2(i)}} b_{i k_{s}}=3(n-1)^{2} .
$$

Substituting values from (3.12), (3.13) in (3.9) we get

$$
X_{i}=n_{2}(i)-3(n-1)^{2} .
$$

Now if $n_{2}(i)=3(n-1)^{2}$ for all $i$ in $H$, then $X_{i}=0$ for all $i$ in $H$. Then it follows from (3.9) that $B$ is a $(0,1)$ matrix which proves (i).

To prove (ii), we note that if $A_{i j_{t}}=1$, then from (3.10), (3.3) and (3.6) it follows

$$
\sum_{t=1}^{n_{1}} b_{i j_{t}}=0 .
$$

But since $b_{i j}=0$ or 1 , this implies $b_{i j_{t}}=0$, and hence from (3.10) it follows that $A_{i j}^{2}=n-2$.

To prove (iii) we note that if $A_{i j}=0, A_{i j}^{2} \neq 0$, then $b_{i j} \neq 0$ and hence $A_{i j}^{2}=2$.

4. THEOREM. If $H$ is a graph satisfying the following properties:

$\left(P_{1}\right)$ The number of vertices is $n^{3}$.

$\left(P_{2}\right) \quad H$ is connected and regular.

$\left(P_{3}\right) \quad n_{2}(x)=3(n-1)^{2}$ for all $x$ in $H$.

$\left(P_{4}\right)$ The distinct eigenvalues of $A(H)$ are $-3, n-3,2 n-3,3(n-1)$. Then, for $n>7, H$ is cubic lattice. 
Proof. From Lemmas (3.1) - (3.3) and the hypothesis $H$ clearly satisfies the following conditions:

$\left(b_{1}\right)$ The number of vertices is $n^{3}$.

$\left(b_{2}\right) \quad H$ is connected and regular of degree $3(n-1)$.

$\left(b_{3}\right) \quad \Delta(x, y)=n-2$ for $d(x, y)=1$.

$\left(b_{4}\right) \quad \Delta(x, y)=2$, for $d(x, y)=2$.

Hence if $n>7, H$ is cubic lattice [6], [4].

Note. It is conjectured that the property $\left(P_{3}\right)$ of the theorem is implied by other properties $\left(P_{1}\right),\left(P_{2}\right),\left(P_{4}\right)$.

It may be pointed out that the main purpose of assuming $\left(P_{3}\right)$ is to prove that $B$ is a $(0,1)$ matrix. If we replace $\left(P_{3}\right)$ by $\left(P_{3}^{\prime}\right)$ and $\left(P_{3}^{\prime \prime}\right)$ as follows:

$\left(P_{3}^{\prime}\right) . \quad H$ is edge-regular, i.e., $\Delta(x, y)=\Delta$ for all $x, y$, such that $\Delta(x, y)=1$,

$$
\left(P_{3}^{\prime \prime}\right) \Delta(x, y)=\text { even, for all } x, y \text {, such that } d(x, y)=2 \text {, }
$$

then it can be shown that $B$ is a $(0,1)$ matrix. The proof goes like this: From $\left(P_{3}^{\prime}\right)$ and (3.3) it follows that $\Delta=n-2$. Substituting value for $\Delta$ in (3.10) and noting $\left(P_{3}^{\prime \prime}\right)$ we get $b_{i j}=0$ if $A_{i j}=1$, and $b_{i j}=$ an integer if $A_{i j}=0$. Again from (3.10) and (3.12) it follows that

$$
\sum_{j=1}^{v} b_{i j}=\sum_{j=1}^{v} b_{i j}^{2}
$$

Thus $B$ is a matrix whose entries are either 0 or integer such that for any row, sum of the elements is equal to the sum of the squares of the elements, but this implies that $B$ is a $(0,1)$ matrix.

Hence we can also state that for $n>7,\left(P_{1}\right),\left(P_{2}\right),\left(P_{3}^{\prime}\right),\left(P_{3}^{\prime \prime}\right),\left(P_{4}\right)$ characterize a cubic lattice graph with characteristic $n$.

\section{REFERENCES}

1. R. C. Bose and R. Laskar, Eigenvalues of the adjacency matrix of tetrahedral graphs, Institute of Statistics Mimeo Series No. 571, University of North Carolina, March 1968.

2. R. C. Bose and D. M. Mesner, On linear associative algebras corresponding to association schemes of partially balanced designs, Ann. Math. Statist. 30 (1959), 21-36. 3. R. C. Bose and T. Shimamoto, Classification and analysis of partially balanced incomplete block designs with two associate classes, J. Amer. Stat. Assn. 47 (1952), 151-184.

4. T. A. Dowling, Note on "A characterization of cubic lattice graphs", J. Combinatorial Theory (to appear)

5. A. J. Hoffman, On the polynomial of a graph Amer. Math. Monthly 70 (1963), 30-36.

6. R. Laskar, A characterization of cubic lattice graphs, J. Combinatorial Theory 3 (1967), 386-401.

Received May 10, 1968. This research was supported by the National Science Foundation Grant No. GP-5790, and the Army Research Office (Durham) Grant No. DA-ARO-D-31-12-G910. 



\section{PACIFIC JOURNAL OF MATHEMATICS}

\section{EDITORS}

H. ROYDEN

Stanford University

Stanford, California

\author{
R. R. Phelps \\ University of Washington \\ Seattle, Washington 98105
}

J. DugundJI

Department of Mathematics

University of Southern California

Los Angeles, California 90007

RICHARD ARENS

University of California

Los Angeles, California 90024

\section{ASSOCIATE EDITORS}

E. F. BECKenbaCH

B. H. NEUMANN

F. WOLF

K. YoSHIDA

\section{SUPPORTING INSTITUTIONS}

\author{
UNIVERSITY OF BRITISH COLUMBIA \\ CALIFORNIA INSTITUTE OF TECHNOLOGY \\ UNIVERSITY OF CALIFORNIA \\ MONTANA STATE UNIVERSITY \\ UNIVERSITY OF NEVADA \\ NEW MEXICO STATE UNIVERSITY \\ OREGON STATE UNIVERSITY \\ UNIVERSITY OF OREGON \\ OSAKA UNIVERSITY \\ UNIVERSITY OF SOUTHERN CALIFORNIA
}

\author{
STANFORD UNIVERSITY \\ UNIVERSITY OF TOKYO \\ UNIVERSITY OF UTAH \\ WASHINGTON STATE UNIVERSITY \\ UNIVERSITY OF WASHINGTON \\ $\stackrel{*}{*} \stackrel{*}{*} \stackrel{*}{*}$ AMERICAN MATHEMATICAL SOCIETY \\ CHEVRON RESEARCH CORPORATION \\ TRW SYSTEMS \\ NAVAL WEAPONS CENTER
}

The Supporting Institutions listed above contribute to the cost of publication of this Journal, but they are not owners or publishers and have no responsibility for its content or policies.

Mathematical papers intended for publication in the Pacific Journal of Mathematics should be in typed form or offset-reproduced, double spaced with large margins. Underline Greek letters in red, German in green, and script in blue. The first paragraph or two must be capable of being used separately as a synopsis of the entire paper. It should not contain references to the bibliography. Manuscripts, in duplicate if possible, may be sent to any one of the four editors. Please classify according to the scheme of Math. Rev. 36, 1539-1546. All other communications to the editors should be addressed to the managing editor, Richard Arens, University of California, Los Angeles, California, 90024.

50 reprints are provided free for each article; additional copies may be obtained at cost in multiples of 50 .

The Pacific Journal of Mathematics is published monthly. Effective with Volume 16 the price per volume (3 numbers) is $\$ 8.00$; single issues, $\$ 3.00$. Special price for current issues to individual faculty members of supporting institutions and to individual members of the American Mathematical Society: $\$ 4.00$ per volume; single issues $\$ 1.50$. Back numbers are available.

Subscriptions, orders for back numbers, and changes of address should be sent to Pacific Journal of Mathematics, 103 Highland Boulevard, Berkeley, California, 94708.

PUBLISHED BY PACIFIC JOURNAL OF MATHEMATICS, A NON-PROFIT CORPORATION

Printed at Kokusai Bunken Insatsusha (International Academic Printing Co., Ltd.), 7-17, Fujimi 2-chome, Chiyoda-ku, Tokyo, Japan. 


\section{Pacific Journal of Mathematics \\ Vol. 29, No. $3 \quad$ July, 1969}

Herbert James Alexander, Extending bounded holomorphic functions from certain subvarieties of a polydisc ...................... 485

Edward T. Cline, On an embedding property of generalized Carter

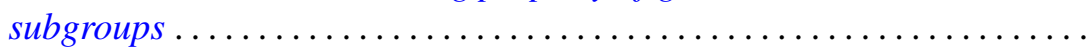

Roger Cuppens, On the decomposition of infinitely divisible characteristic functions with continuous Poisson spectrum. II ...............

William Richard Emerson, Translation kernels on discrete Abelian

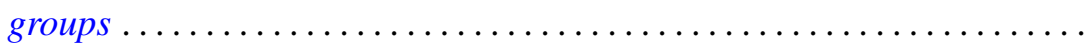

Robert William Gilmer, Jr., Power series rings over a Krull domain ....... 543

Julien O. Hennefeld, The Arens products and an imbedding theorem ...... 551

James Secord Howland, Embedded eigenvalues and virtual poles ........ 565

Bruce Ansgar Jensen, Infinite semigroups whose non-trivial homomorphs are all isomorphic .............................. 583

Michael Joseph Kascic, Jr., Polynomials in linear relations. II .......... 593

J. Gopala Krishna, Maximum term of a power series in one and several

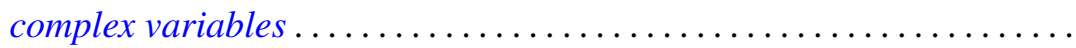

Renu Chakravarti Laskar, Eigenvalues of the adjacency matrix of cubic lattice graphs ...................................

Thomas Anthony Mc Cullough, Rational approximation on certain plane

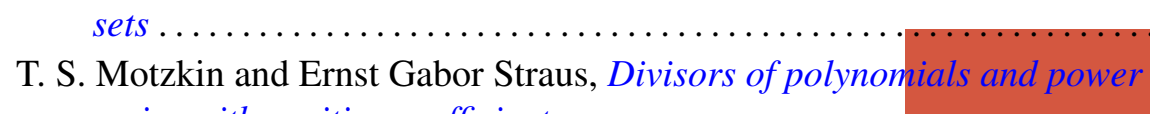
series with positive coefficients .

Graciano de Oliveira, Matrices with prescribed characteristic polynomial and a prescribed submatrix.

Graciano de Oliveira, Matrices with prescribed characteristic polynomial and a prescribed submatrix. II .

Donald Steven Passman, Exceptional 3/2-transitive permutation groups .................................

Grigorios Tsagas, A special deformation of the metric with no negative sectional curvature of a Riemannian space............

Joseph Zaks, Trivially extending decompositions of $E^{n}$ 